\title{
Benign Gastrointestinal Stromal Tumor
}

National Cancer Institute

\section{Source}

National Cancer Institute. Benign Gastrointestinal Stromal Tumor. NCI Thesaurus. Code C53998.

A gastrointestinal stromal tumor that is characterized by a maximum diameter equal or less than $5 \mathrm{~cm}$ (gastric localization), or equal or less than $2 \mathrm{~cm}$ (intestinal localization) and no more than 5 mitotic figures per 50 high power fields. 